A torsion sensor made of a corrugated long period fibre grating

This article has been downloaded from IOPscience. Please scroll down to see the full text article.

2001 Meas. Sci. Technol. 12793

(http://iopscience.iop.org/0957-0233/12/7/307)

The Table of Contents and more related content is available

Download details:

IP Address: 140.112.113.225

The article was downloaded on 13/02/2009 at 09:21

Please note that terms and conditions apply. 


\title{
A torsion sensor made of a corrugated long period fibre grating
}

\author{
L A Wang, C Y Lin and G W Chern \\ Department of Electrical Engineering and Institute of Electro-Optical Engineering, \\ National Taiwan University, Taipei, Taiwan, Republic of China \\ E-mail: lon@ccms.ntu.edu.tw (L A Wang)
}

Received 10 January 2001, in final form 21 March 2001, accepted for publication 3 April 2001

\begin{abstract}
Based on the photoelastic effect, the strain distribution within a corrugated long period fibre grating (LPFG) subjected to tensile stress and twisting will cause a periodic index variation, which may result in resonant couplings between fibre core and cladding modes. The resonance wavelength corresponding to the peak loss is directly sensitive to the torsional angle per unit length; thus a novel torsion sensor can be made from the corrugated long period fibre grating. We also present a phenomenological theory based on the scattering matrix formalism to qualitatively explain the mode coupling behaviour in the corrugated grating.
\end{abstract}

Keywords: strain, torsion, fibre grating, mode coupling, corrugated structure, fibre sensor

\section{Introduction}

Numerous solutions for measuring the applied torque necessary for twisting a shaft have been proposed. In most of these methods one must first obtain the torsion angle by measuring the difference of arc shifts from two position sensors mounted on the shaft; the measured angle is then related to the applied torque. Such sensors, which have generally been developed with optical rotary encoders or magnetic ones, require high precision in the detection of displacement for the torque measurement [1]. Therefore, a sensor that can directly detect the torsion angle instead of making use of two position sensors is attractive.

Grating-based fibre sensors are simple, intrinsic and have all the advantages normally attributed to fibre sensors, such as electrically passive operation, immunity to electromagnetic radiation, high sensitivity and multiplexing capability [2]. For example, a fibre Bragg grating (FBG) has recently been used as a sensor to measure torsion [3]. However, this type of torsion sensor has to be bonded onto the surface of a shaft. When the shaft is twisted, the strain components are induced within the FBG along the fibre axis and the Bragg wavelength will shift accordingly. The amount of torsion can be measured indirectly by detecting the Bragg wavelength shift. Other fibre grating sensors are based on the long-period fibre grating (LPFG), which couples light from the core mode to cladding modes [4], and are more sensitive to the environment. Various applications for sensing temperature, strain, refractive index and bending curvature based on the use of LPFGs have been reported [5-7]. However, when conventional LPFGs are twisted, little index perturbation is induced because the fibre structure is uniform. Thus, no torsion sensor based on a LPFG, which is directly sensitive to the applied torsion, has been reported so far. In this paper, we describe a new type of LPFG made of a corrugated structure over which a periodic strain distribution is created under external mechanical forces such as tensile stress and torsion. Owing to the photoelastic effect, the strain distribution will cause a periodic variation in index and result in mode couplings between the fundamental core mode and the forward-propagating cladding modes. The strain distribution established under mechanical equilibrium will influence the peak transmission loss and the phase-matching condition; thus the corrugated LPFG is sensitive to the external stresses and can act as a novel tensile strain and torsion sensor. We experimentally demonstrate the unique characteristics of a corrugated LPFG suitable as a torsion sensor. We also present a phenomenological theory which is based on the scattering matrix formalism. Since the index perturbations caused by the stress or twist are small, we may expand the scattering coefficients in power series of these small parameters. The physical meaning of these expansion coefficients and their relation to the experimental results are discussed. Under the approximation that the backward reflections are negligible, as is also observed in experiments, a set of forward transfer 


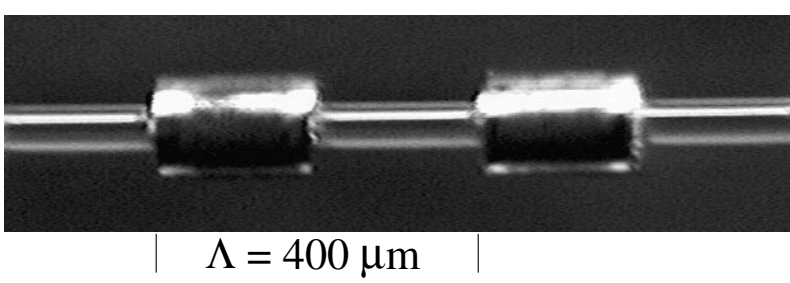

Figure 1. A photomicrograph of a partial section of the corrugated LPFG.

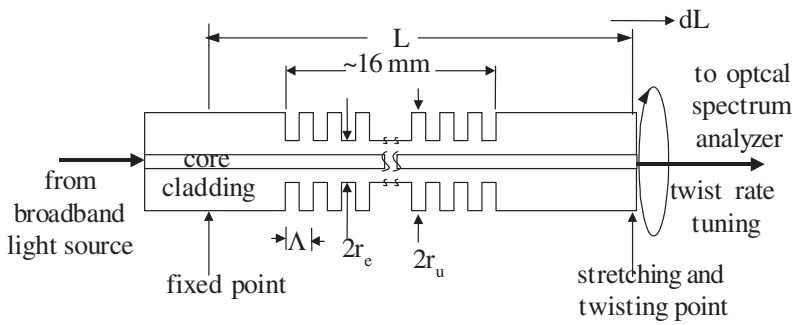

Figure 2. A schematic diagram of a corrugated LPFG and its measurement set-up.

matrices can be used to describe the mode couplings. We also derive a formula for the transmission loss at the phasematched wavelength. The rest of the paper is arranged as follows. In section 2, the experimental results of such sensors for measuring tensile strain and the twist rate are reported. A phenomenological theory is presented in section 3 to explain the principle of coupling between the core and cladding modes through the corrugated structure. Finally, we present our conclusions.

\section{Experiments}

A dispersion-shifted fibre (DSF) with an original cladding radius $r_{u}$ of $62.5 \mu \mathrm{m}$ is used. The corrugated structure is built by etching a pre-patterned fibre using hydrofluoric acid solution [8]. The photomicrograph shown in figure 1 is a partial section of the corrugated LPFG, which has a period of $\Lambda \simeq$ $400 \mu \mathrm{m}$, a total length of $l_{g} \sim 16 \mathrm{~mm}$ and an etched diameter $2 r_{e}$ of about $60 \mu \mathrm{m}$ controlled by the etching time. The experimental set-up for characterizing the corrugated LPFG in sensing tensile strain and torsion is depicted in figure 2. A tensile force and an external torsion torque are applied to induce an average tensile strain $s=\mathrm{d} L / L$ and an average twisting rate $\tau=2 \pi N / L\left(\mathrm{rad} \mathrm{m}^{-1}\right)$, respectively. Here $\mathrm{d} L$ and $N$ are the total elongation and the number of torsional turns over the distance $L$ between the fixed and the stretching points. Since under mechanical equilibrium the induced tensile strain is inversely proportional to the cross-sectional area, we have the following expressions for strains between the unetched and etched regions

$$
s_{e}=\left(r_{u} / r_{e}\right)^{2} s_{u}
$$

where we use the subscripts $u$ and $e$ to denote quantities corresponding to the unetched and etched regions. The total elongation $\mathrm{d} l_{g}$ over the length of the corrugated fibre $l_{g}$ is

$$
\mathrm{d} l_{g}=\frac{l_{g}}{2}\left(s_{u}+s_{e}\right)=\frac{l_{g}}{2}\left[1+\left(\frac{r_{u}}{r_{e}}\right)^{2}\right] s_{u}
$$

The measured mean strain, $s$, over the total length of fibre is

$$
\begin{aligned}
s & \equiv \frac{\mathrm{d} L}{L}=\frac{1}{L}\left[\left(L-\frac{l_{g}}{2}\right) s_{u}+\frac{l_{g}}{2} s_{e}\right] \\
& =\left\{1+\frac{l_{g}}{2 L}\left[\left(\frac{r_{u}}{r_{e}}\right)^{2}-1\right]\right\} s_{u} .
\end{aligned}
$$

The mean strain over the length of corrugated fibre $s_{g} \equiv \mathrm{d} l_{g} / l_{g}$ is thus related to the measured value $s$ as follows:

$$
\begin{aligned}
s_{g} & =\frac{\left(r_{u} / r_{e}\right)^{2}+1}{2+\left(l_{g} / L\right)\left[\left(r_{u} / r_{e}\right)^{2}-1\right]} \frac{\mathrm{d} L}{L} \\
& \equiv Q_{s} s
\end{aligned}
$$

where we introduced a geometry enhancement factor for strain $Q_{s}$. Similarly, for a uniform fibre under fixed torsion, the twisting rate is inversely proportional to the fourth power of the cladding radius; thus we have

$$
\tau_{e}=f\left(r_{u} / r_{e}\right)^{4} \tau_{u}
$$

In the above expression, a factor $f$ is introduced to account for the enhancement due to the effect of the concentration of stress around the discontinuities. Similarly the mean twisting rate $\tau_{g}$ over the length of corrugated fibre is related to the measured value as follows:

$$
\begin{aligned}
\tau_{g} & =\frac{f\left(r_{u} / r_{e}\right)^{4}+1}{2+\left(l_{g} / L\right)\left[f\left(r_{u} / r_{e}\right)^{4}-1\right]} \tau \\
& \equiv Q_{\tau} \tau .
\end{aligned}
$$

A geometry enhancement factor for torsion $Q_{\tau}$ is introduced in the above expression. To have precise control over the adjustment of $\tau$ and $\mathrm{d} L / L, L$ is set as long as $1 \mathrm{~m}$ owing to the limited precision of our mechanical stages. Since the total length $L$ is much larger than the length of corrugated grating $l_{g}$ in this set-up, the strain and twisting rate within the unetched region are almost identical to the measured values,

$$
\begin{gathered}
s_{u} \cong s=\mathrm{d} L / L \\
\tau_{u} \cong \tau=2 \pi N / L \quad\left(L \gg l_{g}\right) .
\end{gathered}
$$

The geometry enhancement factors $Q_{s}$ and $Q_{\tau}$ for the current structure are 2.67 and 9.9, respectively ( $f$ is assumed to be 1 for $Q_{\tau}$ ). A broadband light source and an optical spectrum analyser are used for the measurement of spectral behaviours and power variations.

A typical spectral response of the corrugated LPFG under increasing applied strain $\mathrm{d} L / L$ without twisting is shown in figure 3. One resonant coupling mode resulting from the corrugated LPFG is observed in the wavelength range and its transmission loss at the resonance peak increases with the applied strain. Note that, from figure 3, the peak resonance wavelengths are always obtained at a nearly fixed wavelength regardless of the strain. This characteristic is very distinct from that of the conventional LPFGs which exhibit a considerable amount of wavelength shift during the growth of index modulation [9]. To measure the dependence of the transmission loss on tensile strain, we increase the strain by a small step ( $\simeq 45 \mu$ strain) and measure the ratio of the transmitted power $P_{t}$ to the initial power $P_{0}$ at the peak resonance wavelength $(\simeq 1279 \mathrm{~nm})$. The measured result is 


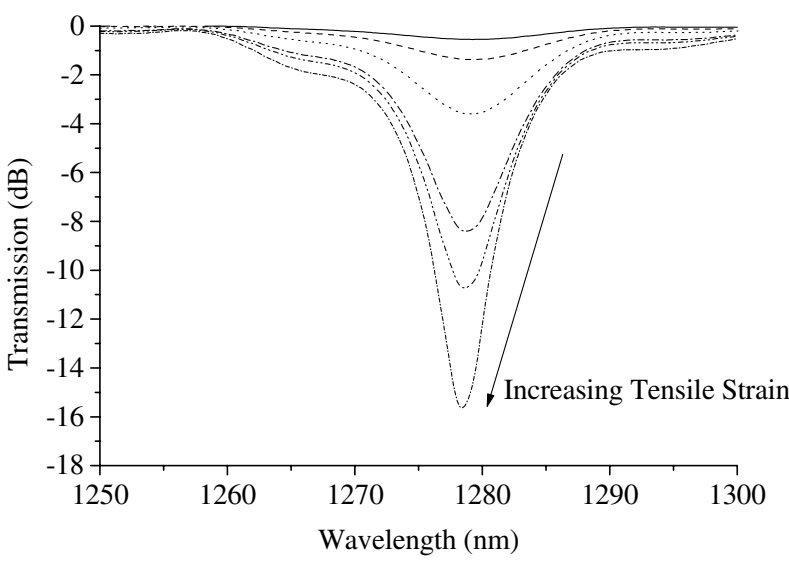

Figure 3. The evolution of transmission spectra of a corrugated LPFG when purely tensile strains are applied.

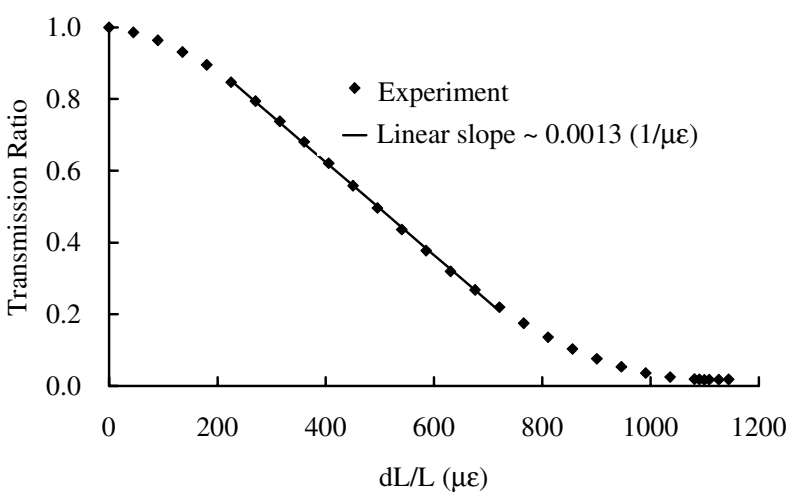

(a)

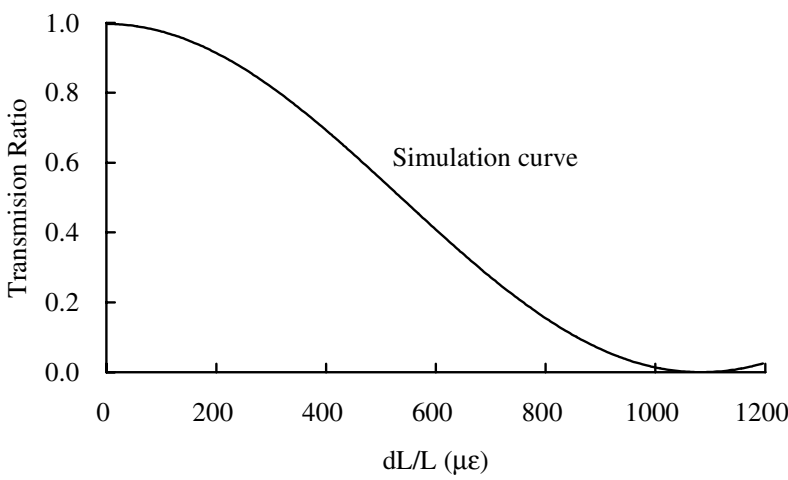

(b)

Figure 4. (a) The measured peak transmission loss versus applied tensile strain of the corrugated LPFGs. (b) The calculated dependence of the transmission loss on the applied tensile strain obtained by using equation (13) in section 3 .

shown in figure 4. A strain gauge factor, an indicator of the sensitivity of the sensor, is defined as $\left(1-P_{t} / P_{0}\right) /(\mathrm{d} L / L)$. The measured strain gauge factor of the corrugated LPFG sensor is found to be about 1300 in the linear region. Since the strain gauge factor is dependent on the geometry parameters which are determined by the fabrication and experimental setup (cf equation (2)), it should be normalized by the geometry enhancement factor $Q_{s}$. A value of about 440 is obtained for the corrugated LPFG, which is even more sensitive than those reported before $[5,9]$.

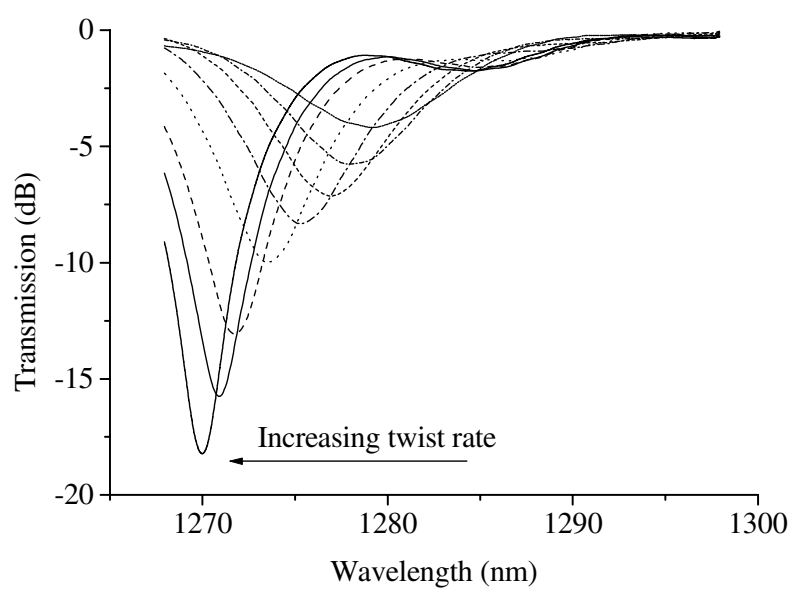

(a)

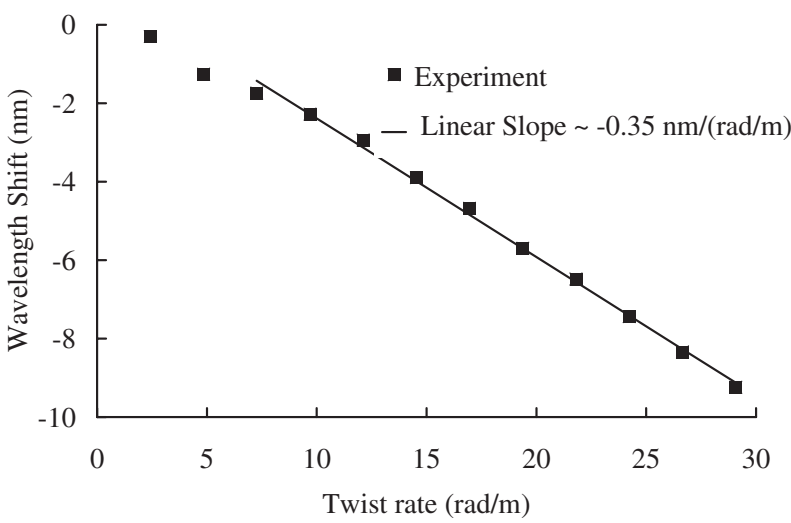

(b)

Figure 5. (a) The evolution of transmission spectra of the torsion sensor. (b) The measured peak resonant wavelength versus applied twisting rates

From figure 3 , it can be seen that there is very weak resonant mode coupling for a corrugated LPFG when neither tensile strain nor torsion is applied. This implies that, to characterize the shift in resonance wavelength owing to the effect of torsion, the corrugated LPFG needs to be loaded with a pre-tensile strain for an initial resonant mode coupling. In measurements, therefore, the fibre is applied with a fixed pretensile strain ( $\simeq 600 \mu$ strain) and twisted by rotating one end with the other end fixed. A typical spectral evolution for the corrugated LPFG with increasing twisting rate is shown in figure 5(a). Apart from the change in the resonant loss, the resonance wavelength shifts toward the shorter wavelength side. For comparison, we also do a similar torsion experiment on a conventional LPFG and observe no obvious change in transmission spectrum even when the twisting rate is doubled. Therefore, such a unique characteristic making this system applicable as a torsion sensor can mainly be attributed to the corrugated structure.

As shown in figure 5(b), by measuring the peak resonance wavelength in figure $5(a)$, the intrinsic sensitivity of the torsion sensor is found to be about $0.036 \mathrm{~nm} \mathrm{rad}{ }^{-1} \mathrm{~m}$ after being normalized with respect to the geometry enhancement factor $Q_{\tau}$ (cf equation (4)). In figure 3 , it has been shown that the peak resonance wavelengths are always obtained at a nearly fixed wavelength under purely tensile strain. Now, 


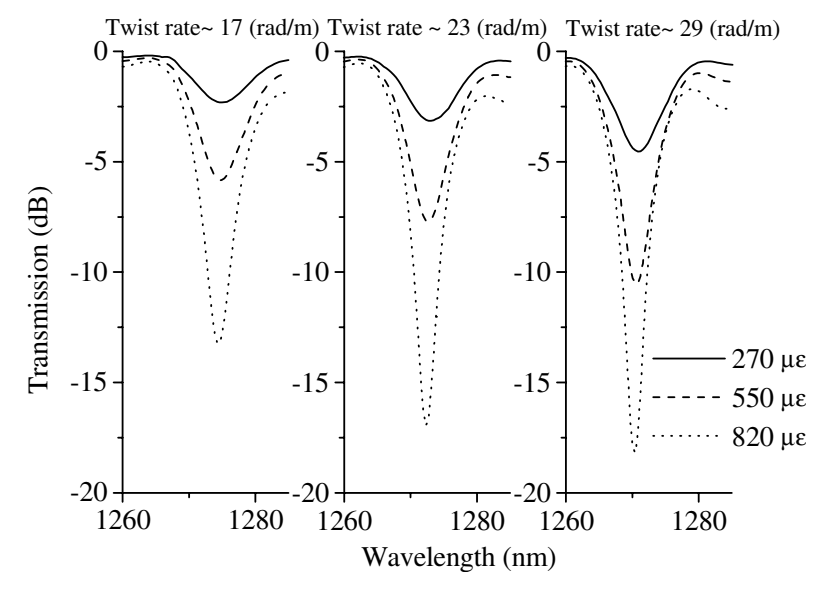

Figure 6. The evolution of transmission spectra of the corrugated LPFG when three different twisting rates combined with increasing tensile strains are applied.

the evolutions of transmission spectra of the LPFG at three different fixed twisting rates with increasing tensile force are shown in figure 6 . It is seen that the shift in resonance wavelength is almost entirely determined by the twisting rate regardless of the amount of tensile strain.

The measured temperature dependence of the fibre sensor is less than $+0.05 \mathrm{~nm}{ }^{\circ} \mathrm{C}^{-1}$, which is similar to that of a conventional LPFG [5], implying that the thermal stresses induced by a change in temperature have little dependence on the diameter of the fibre [10]. Note that, for practical applications, it is necessary to compensate for the effect of the ambient temperature. Since a conventional uniform fibre grating is insensitive to torsional stress, it can function as a temperature sensor when it is combined with our corrugated LPFG to make one fibre device. Thus, the wavelength shift resulting from a pure twist can be separated from the thermal one.

In principle, the torsion fibre sensor in line form may be implemented in the following ways. The torsion shaft to be measured is implanted with the corrugated fibre sensor which is free of contact with the shaft in order to avoid unnecessary stresses. Then the fibre is fixed with pre-tensile strain on both ends of the shaft. In this way, the twisting rate of the shaft is the same as that of the fibre sensor. Another way is to fix the prestressed fibre device with the centres of two stiff discs at both ends. Both discs are coaxial with the shaft. One disc is fixed, but the other is connected to the rotating shaft. When the shaft is twisted, the fibre device is also subjected to the same torsion angle at the connection and acts as a torsion angle sensor. So, the twisting rate of the shaft can be detected as the measured one of the fibre device multiplied by the ratio of the length of the shaft to that of the fibre device. Since the sensing length of the fibre device may be just a few centimetres, which is much less than that of the shaft in most cases, the sensitivity of our torsion sensor constructed in the latter way should be great enough for engineering purposes.

It should be mentioned that the measurement results on the sensitivities to strain and the twisting rate are reproducible, Also, no measurable degradation is detected within the measuring ranges.

\section{A phenomenological theory}

Conventionally, the coupled-mode theory (CMT) $[4,11]$ is used to describe the interactions between waveguide modes when there is a longitudinal perturbation within the guide. However, in the case of a corrugated LPFG, the etching depth is so large that the corrugated structure cannot be regarded as a perturbation. Owing to the discontinuities of the guiding structure between the etched and unetched regions, a wavefield incident on the hetero-interface would excite many other guiding as well as radiation modes. We shall use the scattering matrix method $[12,13]$ to describe mode couplings at these discontinuities. Let the scattering coefficients $r_{i j}$ and $t_{i j}$ be the amplitude ratios of the backward- and forward-propagating mode $j$ excited by a wavefield of mode $i$ incident on the heterointerface from the unetched to the etched region. The periods of the grating are designed to allow resonant couplings between the fundamental core mode and cladding modes of the first few radial orders; for the fibre used in our experiment these modes are relatively centrally confined. For the application of the corrugated LPFGs as tensile and torsion sensors, we mainly consider resonant two-mode couplings between the fundamental core mode and a chosen phase-matched cladding mode. From our experimental measurement, the reflection coefficients are much smaller than the transmission ones for the modes under consideration, thus we may neglect them in the following discussion. Similarly, we let $t_{i j}^{\prime}$ be the corresponding transmission coefficients for incidence from the etched region to the unetched region. The exact calculation of the transmission coefficients is sophisticated and usually requires solving large linear equations or other methods of simplification. However, in the following, we shall develop a phenomenological theory to describe the resonant coupling behaviour of such a corrugated LPFG.

Because the corrugated LPFG is under torsion, we use the following dimensionless parameter to characterize the twisting rate $\tau$.

$$
\varpi=\tau \Lambda^{(0)}=2 \pi N \Lambda^{(0)} / L
$$

where $\Lambda^{(0)}$ is the period of the unstrained grating. From equations (1) and (3), the strains and twisting rates within the unetched and etched regions are different due to the difference in radius of cladding. Through the photoelastic effect, different index perturbations are induced within these two regions. The modal properties of these two regions are thus perturbed according to the corresponding index changes and the resultant mode mismatch will also contribute to mode couplings apart from that due to the discontinuous interface. The transmission coefficients are thus dependent on the applied stress, either tensile force or torsion, and we may expand the transmission coefficients perturbatively in Taylor series of the corresponding parameters as follows $(i, j=\mathrm{co}, \mathrm{cl})$ :

$$
\begin{aligned}
t_{i j}(s, \varpi)=t_{i j}^{(0)}+a_{i j}^{(1)} s & +a_{i j}^{(2)} s^{2}+\cdots \\
+b_{i j}^{(1)} \varpi & +b_{i j}^{(2)} \varpi^{2}+\cdots \\
& +c_{i j}^{(2)} \varpi s+\cdots
\end{aligned}
$$

where $a_{i j}$ and $b_{i j}$ are the corresponding expansion parameters for the tensile strain and twisting rate, whereas $c_{i j}$ accounts for the combined effect of twisting and strain on the transmission 
coefficient. The importance of this cross term is evident from the results in figure 6 , which shows the dependence of the transmission loss on the applied strain for three twisting rates. It is expected that all these expansion coefficients except the zeroth term decrease as the cladding ratio $r_{u} / r_{e}$ tends to unity, corresponding to a uniform fibre structure. The zeroth order terms $t_{i j}^{(0)}$ represent the intrinsic transmission coefficients of the corrugated structure, while the higher order terms are due to the index perturbations caused by the induced difference in strain. As described in section 2, the measured transmission loss of the corrugated LPFG without any external stress is fairly small; therefore, we have the following properties for the zeroth-order terms:

$$
t_{c o-c o}^{(0)} \cong t_{c l-c l}^{(0)} \cong 1 \quad t_{c o-c l}^{(0)}, t_{c l-c o}^{(0)} \ll t_{c o-c o}^{(0)}, t_{c l-c l}^{(0)} .
$$

This can be attributed to the similarities of the coupled modes under consideration, i.e. the mode fields belonging to the two regions have similar profiles, nodal points, etc. In addition, from the perturbation theory, the first-order terms of self-transmission coefficients are also very small; this is because they are related to the zeroth-order terms of the crosstransmission coefficients $t_{c o-c l}^{(0)}$ and $t_{c l-c o}^{(0)}$ from the perturbation theory [15]. We shall first discuss the dependence of the transmission loss on the external stress. As described in section 2, the main contribution to the loss is from the difference in tensile strain between the two regions. Thus, for the sake of clarity, we shall consider the effects of tensile strain on the transmission loss. Under such an approximation, we have for the transmission coefficients

$$
\begin{gathered}
t_{c o-c o} \cong t_{c o-c o}^{(0)}+a_{c o-c o}^{(2)} s^{2} \equiv t_{c o}\left(1-\alpha_{c o} s^{2}\right) \\
t_{c l-c l} \cong t_{c l-c l}^{(0)}+a_{c l-c l}^{(2)} s^{2} \equiv t_{c l}\left(1-\alpha_{c l} s^{2}\right) \\
t_{c l-c o} \cong a_{c l-c o}^{(1)} s \equiv \gamma s \\
t_{c o-c l} \cong a_{c o-c l}^{(1)} s \equiv-\gamma^{\prime} s .
\end{gathered}
$$

Here we keep terms up to the first order in strain $s$; the inclusion of the second-order terms $\alpha s^{2}$ is due to field renormalization of perturbation and is required for the conservation of power consistently with the second-order expansion [15]. By neglecting reflections, the transmission coefficients $t_{i j}^{\prime}$ are the transpose of $t_{i j}$ [14], i.e.

$$
t_{i j}^{\prime}=t_{j i} \quad(i, j=\mathrm{co}, \mathrm{cl}) .
$$

On the basis of these results, a set of forward transfer matrices $[14,15]$ can be used to describe the coupling behaviour of the corrugated LPFG as detailed in the appendix. We now consider the phase-matching condition. Let the propagation constants of mode $j$ in the unetched and etched regions be $\beta_{u, j}$ and $\beta_{e, j}$, respectively. The optical phase differences (OPDs) between the core and cladding modes through the unetched and etched regions are denoted $\Delta_{u}$ and $\Delta_{e}$, respectively, and are given as follows:

$$
\Delta_{u}=\left(\beta_{u, c o}-\beta_{u, c l}\right) \Lambda_{u} \quad \Delta_{e}=\left(\beta_{e, c o}-\beta_{e, c l}\right) \Lambda_{e}
$$

The phase-matching condition, which corresponds to the inphase couplings through each period, is given by [15]

$$
\Delta=\Delta_{u}+\Delta_{e}=2 \pi
$$

where $\Delta$ is the total OPD through a period, which is obviously also a function of the applied tensile strain $s$ and the twisting rate $\varpi$. Assuming that $\Lambda_{u}=\Lambda_{e}=\Lambda / 2$, as used in our experiment, the phase-matching condition can be written as

$$
\bar{\beta}_{c o}-\bar{\beta}_{c l}-2 \pi / \Lambda=0
$$

where $\bar{\beta}_{j}=\left(\beta_{u, j}+\beta_{e, j}\right) / 2(j=\mathrm{co}, \mathrm{cl})$ is the averaged propagation constant of mode $j$. As derived in the appendix, we have the following formula for the phasematched transmission loss:

$$
T(s)=\left|\frac{A(s) \sin [N \phi(s)]-\sin [(N-1) \phi(s)]}{\sin [\phi(s)]}\right|^{2}
$$

where the functions $A(s)$ and $\phi(s)$ are given in the appendix. In figure $4(b)$, we show the calculated dependence of the transmission loss on the applied tensile strain by using the above expression for comparison with the experiment; qualitative agreement between them can be seen.

As shown in section 2, the resonance wavelength is strongly influenced by the applied twisting rate. In order to investigate the dependence of the phase-matching condition on the applied stresses, we may also expand the OPD, $\Delta$, into a Taylor series by using the small parameters $s$ and $\varpi$ :

$$
\Delta \cong \Delta^{(0)}+\Delta^{(a)} s+\Delta^{(b)} \varpi
$$

where $\Delta^{(a)}$ and $\Delta^{(b)}$ are the first-order expansion coefficients of the tensile strain and torsion, respectively. There are two contributions to the strain coefficient $\Delta^{(a)}$. One is the difference between corrections of propagation constants due to the strain-induced changes in index for the two modes, which are also termed self-couplings in the formulation of coupled-mode theory. In the case of a corrugated LPFG, the self-coupling constant of the cladding mode due to the straininduced index perturbation is comparable to that of the core mode. This is because the index perturbation is not confined exclusively to the core of the fibre as in conventional photoinduced LPFGs [8]. The other contribution to $\Delta^{(a)}$ is the geometrical elongation of the period. As can be seen from the transmission spectra in section 2 , the resonance wavelength hardly shifts as the strain is increased. This indicates that the first-order term $\Delta^{(a)}$ is very small, which can be attributed to the fact that these two contributions cancel out.

We are now considering the influence of torque on the phase-matching condition. For twist operation, the period remains almost fixed, while the twisting-induced change in index will modify the corresponding transmission coefficients and propagation constants. From the experiments, it is shown that twisting will contribute equally to the cross-transmission coefficients $t_{c o-c l}$ and $t_{c l-c o}$, which also increases the resonance loss as shown in figure $5(a)$. This contribution to the transmission loss is included in the coefficients $b_{i j}$ and $c_{i j}$ cross term in the expansion of the transmission coefficients $t_{i j}$ and can be treated similarly to the case of tensile stress. In addition, the twisting will significantly modify the phase-matching condition. Since the period can be regarded as unchanged for a given tensile strain, the corresponding expansion coefficient corresponding to twisting is given as

$$
\varpi \Delta^{(b)}=\left(\Delta \beta_{u, c o}-\Delta \beta_{u, c l}\right) \Lambda_{u}+\left(\Delta \beta_{e, c o}-\Delta \beta_{e, c l}\right) \Lambda_{e} .
$$


Here $\Lambda_{u}$ and $\Lambda_{e}$ are the lengths of the two regions under a fixed tensile strain. The correction to the propagation constant is related to the corresponding self-coupling constant by

$$
\Delta \beta_{r, j}=\varpi \kappa_{r, j} \quad(r=u, e ; j=\mathrm{co}, \mathrm{cl})
$$

where we have defined $\kappa_{r, c o}$ and $\kappa_{r, c l}$ as the twisting-induced self-coupling constants of the core and cladding modes in region $r$, respectively. As a result, the expansion coefficient $\Delta^{(b)}$ can be expressed as

$$
\begin{aligned}
\Delta^{(b)} & =\left(\kappa_{u, c o}-\kappa_{u, c l}\right) \Lambda_{u}+\left(\kappa_{e, c o}-\kappa_{e, c l}\right) \Lambda_{e} \\
& \equiv\left(\bar{\kappa}_{c o}-\bar{\kappa}_{c l}\right) \Lambda
\end{aligned}
$$

where we have defined the averaged self-coupling constants over the period. Owing to the discontinuities between the etched and unetched regions, the twisting-induced strains are mainly distributed in the cladding region, so we have $\bar{\kappa}_{c l}>\bar{\kappa}_{c o}$. By substituting (14) and (15) into (11), we have for the resonance wavelength

$$
\lambda_{\text {res }}=\frac{\left(\bar{n}_{c o}-\bar{n}_{c l}\right) \Lambda}{1+\varpi\left(\bar{\kappa}_{c l}-\bar{\kappa}_{c o}\right) \Lambda /(2 \pi)}
$$

where $\bar{n}_{c o}$ and $\bar{n}_{c l}$ are the average effective indices of core and cladding modes in the two regions, respectively. As can be seen from the above expression, the resonance wavelength will shift towards the shorter side when the corrugated LPFG is subjected to twisting. From the above discussions of $\Delta^{(a)}$ and $\Delta^{(b)}$, it can be concluded that the coefficient $\left|\Delta^{(b)}\right|$ is much larger than $\Delta^{(a)}$. Thus, from equation (14), the shift in resonance wavelength resulting from the change of OPD, $\Delta$, is dominated by the twisting rate even when twisting is combined with increasing tensile strain, as shown in figure 6 .

\section{Conclusion}

By using the corrugated fibre structure and the photoelastic effect, besides having a tensile strain fibre sensor, we have developed a new technique for making a torsion fibre sensor whose peak resonance wavelength decreases with the applied torsional angle. To the best of our knowledge, this is the first torsion sensor based on the direct detection of the wavelength shift uniquely possessed by the corrugated LPFGs in line form with the advantages of micro-element, electrically passive operation and immunity to electromagnetic radiation. We also present a phenomenological theory, which is based on the scattering matrix formalism, to explain the coupling behaviour between the core and cladding modes in the corrugated fibre with discontinuities of the guiding structure.

\section{Acknowledgments}

The author are grateful for the support in part by the National Science Council Taiwan, ROC, under the contract NSC 892215-E-002-062 and by the Education Ministry under the contract 89-E-FA06-2-4-7 in Taiwan, ROC.

\section{Appendix}

We will use a transfer-matrix approach to describe the mode couplings through the corrugated LPFG using the transmission coefficients outlined in section 3 . Here we consider resonant two-mode coupling and neglect the contributions of backwardpropagating modes. We use a column vector to represent the amplitudes of the core and cladding modes as $\boldsymbol{A}_{r}=$ $\left[A_{r, c o} A_{r, c l}\right]^{T}(r=u, e)$. Then the couplings of the amplitudes when the wavefield crosses the interface from the unetched to the etched region can be expressed as $\boldsymbol{A}_{e}=\mathbf{F}_{1}(s) \boldsymbol{A}_{u}$ where the interface matrix is given by

$$
\mathbf{F}_{1}(s)=\left[\begin{array}{cc}
t_{c o}\left(1-\alpha_{c o} s^{2}\right) & \gamma s \\
-\gamma^{\prime} s & t_{c l}\left(1-\alpha_{c l} s^{2}\right)
\end{array}\right]
$$

and similarly for crossing from the etched to the unetched region, we have with $\boldsymbol{A}_{u}=\mathbf{F}_{2}(s) \boldsymbol{A}_{e}$

$$
\mathbf{F}_{2}(s)=\left[\begin{array}{cc}
t_{c o}\left(1-\alpha_{c o} s^{2}\right) & -\gamma^{\prime} s \\
\gamma s & t_{c l}\left(1-\alpha_{c l} s^{2}\right)
\end{array}\right] .
$$

For free propagation through the separate regions, the amplitudes only have a phase shift and can be described by two phase-shift matrices as

$$
\mathbf{P}_{u}(s)=\left[\begin{array}{cc}
\exp \left(\mathrm{i} \Delta_{u} / 2\right) & 0 \\
0 & \exp \left(-\mathrm{i} \Delta_{u} / 2\right)
\end{array}\right]
$$

for the unetched region and

$$
\mathbf{P}_{e}(s)=\left[\begin{array}{cc}
\exp \left(\mathrm{i} \Delta_{e} / 2\right) & 0 \\
0 & \exp \left(-\mathrm{i} \Delta_{e} / 2\right)
\end{array}\right]
$$

for the etched region; $\Delta_{u}$ and $\Delta_{e}$ are given in equation (10). The transfer matrix of a unit period is given by the following product:

$$
\mathbf{F}=\mathbf{F}_{1} \mathbf{P}_{u} \mathbf{F}_{2} \mathbf{P}_{e}
$$

Under the phase-matching condition (11), the phase differences of the two regions can be expressed as

$$
\Delta_{u}=\pi+\varepsilon \quad \Delta_{e}=\pi-\varepsilon
$$

where $\varepsilon \ll \pi$ representing the difference between the two OPDs under the phase-matching condition. Then the phase matrices take the following forms:

$$
\mathbf{P}_{u}=\left[\begin{array}{cc}
\mathrm{e}^{\mathrm{i} \varepsilon / 2} & 0 \\
0 & -\mathrm{e}^{-\mathrm{i} \varepsilon / 2}
\end{array}\right]
$$

and

$$
\mathbf{P}_{e}=\left[\begin{array}{cc}
\mathrm{e}^{-\mathrm{i} \varepsilon / 2} & 0 \\
0 & -\mathrm{e}^{\mathrm{i} \varepsilon / 2}
\end{array}\right]
$$

By substituting these expressions into (A5), we obtain the transfer matrix of a unit period

$$
\mathbf{F}(s)=\left[\begin{array}{ll}
A(s) & B(s) \\
C(s) & D(s)
\end{array}\right]
$$

with the following matrix elements

$$
\begin{gathered}
A(s)=t_{c o}^{2}-s^{2}\left(2 \alpha_{c o} t_{c o}^{2}+\gamma^{2} \mathrm{e}^{\mathrm{i} \varepsilon}\right) \\
B(s)=+s\left(t_{c o} \gamma^{\prime} \mathrm{e}^{\mathrm{i} \varepsilon}+t_{c l} \gamma\right)
\end{gathered}
$$




$$
\begin{gathered}
C(s)=-s\left(t_{c o} \gamma^{\prime}+t_{c l} \gamma \mathrm{e}^{-\mathrm{i} \varepsilon}\right) \\
D(s)=t_{c l}^{2}-s^{2}\left(2 \alpha_{c l} t_{c l}^{2}+\gamma^{\prime 2} \mathrm{e}^{\mathrm{i} \varepsilon}\right) .
\end{gathered}
$$

For a corrugated LPFG with periods, the total transfer matrix is given by the well known Chebyshev identity $[15,16]$

$$
\mathbf{F}^{N}=\left[\begin{array}{cc}
\frac{A \sin (N \phi)-\sin [(N-1) \phi]}{\sin (\phi)} & B \frac{\sin (N \phi)}{\sin (\phi)} \\
C \frac{\sin (N \phi)}{\sin (\phi)} & \frac{D \sin (N \phi)-\sin [(N-1) \phi]}{\sin (\phi)}
\end{array}\right]
$$

where the parameter $\phi(s)$ is given by

$$
\phi(s)=\cos ^{-1}\left(\frac{A(s)+D(s)}{2}\right) .
$$

The core transmission amplitude is given from (A10) by the matrix element $\left[\mathbf{F}^{N}\right]_{11}$ which is related to the transmission loss of the corrugated LPFG.

\section{References}

[1] Lemarquand V 1999 Synthesis study of magnetic torque sensors IEEE Trans. Magn. 35 4503-10

[2] Kersey A D, Davis M A, Patrick H J, LeBlanc M, Koo K P, Askins C G, Putnam M A and Friebele E J 1997 Fiber grating sensors J. Lightwave Technol. 15 1442-61

[3] Tian X and Tao X 2000 Torsion measurement by using FBG sensors Proc. SPIE 4077 154-64

[4] Erdogan T 1997 Cladding-mode resonances in short- and long-period fibre grating filters J. Opt. Soc. Am. A 14 1760-73
[5] Bhatia V and Vengsarkar A M 1996 Optical fibre long-period grating sensors Opt. Lett. 21 692-4

[6] Patrick H J, Chang C C and Vohra S T 1998 Long period fibre gratings for structural bend sensing Electron Lett. 34 1773-5

[7] Liu Y, Zhang L, Williams J A R and Bennion I 2000 Optical bend sensor based on measurement of resonance mode splitting of long-period fibre grating Photon. Technol. Lett. 12 531-3

[8] Lin C Y and Wang L A 1999 Loss-tunable long period fibre grating made from etched corrugation structure Electron Lett. 35 1872-3

[9] Grubsky V and Feinberg J 2000 Long-period fibre gratings with variable coupling for real-time sensing applications Opt. Lett. $25203-5$

[10] Song M, Lee B, Lee S B and Choi S S 1997 Interferometric temperature-insensitive strain measurement with different-diameter fibre Bragg gratings Opt. Lett. 22 790-2

[11] Marcuse D 1991 Theory of Dielectric Optical Waveguide (Boston: Academic)

[12] Collin R E 1992 Foundations for Microwave Engineering (New York: McGraw-Hill)

[13] Chu T S and Itoh T 1986 Generalized scattering matrix method for analysis of cascaded and offset microstrip step discontinuities IEEE Trans. Microwave Theory Tech. 34 $280-4$

[14] Huang W P and Hong J 1992 A transfer matrix approach based on local normal modes for coupled waveguides with periodic perturbations J. Lightwave Technol. 10 1367-74

[15] Chern G W and Wang L A 1999 Transfer-matrix method based on perturbation expansion for periodic and quasi-periodic binary long-period gratings J. Opt. Soc. Am. A 16 2675-89

[16] Yeh P 1998 Optical Waves in Layered Media (New York: Wiley) 